\title{
Supernova Nucleosynthesis with Neutrino Processes: Dependence of Fluorine abundance on Stellar Mass, Explosion Energy and Metallicity
}

\author{
Natsuko Izutani, Hideyuki Umeda, and Takashi Yoshida
}

Department of Astronomy, School of Science, University of Tokyo, Bunkyo-ku, Tokyo, 113-0033

email: izutani@astron.s.u-tokyo.ac.jp

\begin{abstract}
We investigate the effects of neutrino-nucleus interactions on the production of Fluorine during normal supernovae and hypernovae, and discuss stellar mass, metallicity and explosion energy dependence of $[\mathrm{F} / \mathrm{Fe}, \mathrm{Ne}, \mathrm{O}]$. We find the clear trend of $[\mathrm{F} / \mathrm{Fe}, \mathrm{O}, \mathrm{Ne}]$ with stellar mass and explosion energy, while no clear trend with metallicity. This trend of $[\mathrm{F} / \mathrm{O}]$ can be used to constrain the contributed stellar mass by comparing with the observational abundance.
\end{abstract}

Keywords. neutrinos, nuclear reactions, nucleosynthesis, abundances, supernova: general

\section{Introduction}

The interaction of the neutrinos with matter and the effects on the nucleosynthesis have only been discussed for a few models (e.g., Woosley et al. 1990; Woosley \& Weaver 1995; Yoshida et al. 2004; Heger et al. 2005; Yoshida et al. 2008; Nakamura et al. 2010). The $\nu$-process does not affect the yields of major elements such as Fe and $\alpha$ elements, but it will increase those of some elements such as B, F, K, Sc, V, and Mn. In this paper, we focus on the effect of the $\nu$-process on $\mathrm{F}$ during normal supernova (SN) and hypernova (HN) explosions, and discuss stellar mass, metallicity, and explosion energy dependence of $[\mathrm{F} / \mathrm{Fe}, \mathrm{O}, \mathrm{Ne}]$.

\section{Model \& Method}

We calculate the nucleosynthesis for core-collapse SNe with progenitor masses of $M=$ 15,25 , and $50 M_{\odot}$ and initial metallicities of $Z=0,0.004$, and 0.02 for normal SNe and HNe. The explosion energy is set to be $1 \times 10^{51}$ ergs for normal SNe, $10 \times 10^{51}$ and $40 \times 10^{51}$ ergs for HNe with $M_{\mathrm{MS}}=25$ and $50 M_{\odot}$, respectively. For normal SNe, the mass cut is set to meet the observed iron mass of $0.07 M_{\odot}$. For HNe, the parameters of mixing fallback models are determined to get $[\mathrm{O} / \mathrm{Fe}]=0.5$. The nuclear network includes 809 species up to ${ }^{121} \mathrm{Pd}$ (Izutani et al. 2009, Izutani \& Umeda 2010). We adopt the $\nu$ process up to ${ }^{80} \mathrm{Kr}$ as in Yoshida et al. (2008). The neutrino luminosity is assumed to be uniformly partitioned among the neutrino flavors, and decrease exponentially in time with a timescale of $3 \mathrm{~s}$. The total neutrino energy is set to be $E_{\nu}=3$ and $9 \times 10^{53}$ ergs. The neutrino energy spectra are assumed to be Fermi-Dirac distributions with zero chemical potentials. The temperatures of $\nu_{\mu, \tau}, \bar{\nu}_{\mu, \tau}$ and $\nu_{\mathrm{e}}, \bar{\nu}_{\mathrm{e}}$ are set to be $T_{\nu}=6$ and $4 \mathrm{MeV} / k$, respectively. 

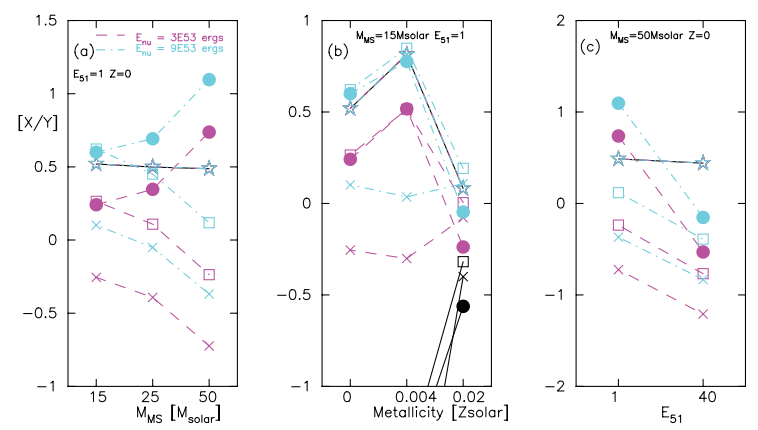

Figure 1. $[\mathrm{F} / \mathrm{Fe}, \mathrm{O}, \mathrm{Ne}]$ (filled circles, open squares, and crosses) and $[\mathrm{Ne} / \mathrm{O}]$ (stars) in the models without $\nu$-processes (black solid lines), with $\nu$-processes of $E_{\nu}=3$ and $9 \times 10^{53}$ ergs (magenta dashed lines and cyan dot-dashed lines). (a) Stellar mass dependence ([X/Y $]$ in the models with $M_{\mathrm{MS}}=15,25,50 M_{\odot}, Z=0$ and $\left.E_{51}=1\right)$ (b) Metallicity dependence ([X/Y] in the models with $Z=0,0.004,0.02, M_{\mathrm{MS}}=15 M_{\odot}$ and $\left.E_{51}=1\right)(\mathrm{c}) E_{51}$ dependence ([X/Y $]$ in the models with $M_{\mathrm{MS}}=50 M_{\odot}, Z=0, E_{51}=1$ and 40 .

\section{Results and Discussion}

With the $\nu$-process, ${ }^{19} \mathrm{~F}$ is produced in the $\mathrm{O} / \mathrm{Ne}$-enriched region through ${ }^{20} \mathrm{Ne}(\nu$, $\left.\nu^{\prime} \mathrm{p}\right)^{19} \mathrm{~F}$. Figure 1 (a) shows mass dependence of $[\mathrm{F} / \mathrm{Fe}, \mathrm{O}, \mathrm{Ne}]$ and $[\mathrm{Ne} / \mathrm{O}]$ in $\mathrm{Z}=0$ star $\mathrm{SNe}$. $[\mathrm{Ne} / \mathrm{O}]$ is about 0.5 in these models. Without the $\nu$-processes, $[\mathrm{F} / \mathrm{Fe}, \mathrm{O}, \mathrm{Ne}]$ are $\sim-5$. With the $\nu$-processes, $[\mathrm{F} / \mathrm{Fe}, \mathrm{O}, \mathrm{Ne}]$ range from -1 to 1 . $[\mathrm{F} / \mathrm{Fe}]$ is higher for more massive stars because of the larger $\mathrm{O} / \mathrm{Ne}$-enriched region. By contrast, $[\mathrm{F} / \mathrm{O}, \mathrm{Ne}]$ are lower for more massive stars because the radius of the $\mathrm{O} / \mathrm{Ne}$-enriched region is larger, and the neutrino flux becomes smaller. Figure 1(b) shows metallicity dependence of $[\mathrm{F} / \mathrm{Fe}, \mathrm{O}, \mathrm{Ne}]$ and $[\mathrm{Ne} / \mathrm{O}]$ in $15 M_{\odot} \mathrm{SNe} .[\mathrm{Ne} / \mathrm{O}]$ is different between these models, though it is not clear whether this trend of $[\mathrm{Ne} / \mathrm{O}]$ is due to metallicity or not. With the $\nu$-processes, the F yield is increased by a factor of $\sim 10$ and 1000 for $Z=0.02$ and 0 , respectively. There is no clear trend of $[\mathrm{F} / \mathrm{Fe}, \mathrm{O}, \mathrm{Ne}]$ with metallicity. Figure 1 (c) shows $E_{\exp }$ dependence of [F/Fe,O,Ne] and $[\mathrm{Ne} / \mathrm{O}]$ in $Z=050 M_{\odot}$ explosions. [Ne/O] is about 0.5 in these models. With the $\nu$ processes, $[\mathrm{F} / \mathrm{Fe}, \mathrm{O}, \mathrm{Ne}]$ range from -1 to 1 . $[\mathrm{F} / \mathrm{O}, \mathrm{Ne}]$ are lower in the $\mathrm{HN}$ model. In the $\mathrm{HN}$ model, the shock wave reaches the $\mathrm{O} / \mathrm{Ne}$-enriched region earlier, and the region expands earlier, which causes smaller neutrino flux. $[\mathrm{F} / \mathrm{Fe}]$ is also lower in the HN model, which is caused by both the larger mass of $\mathrm{Fe}$ and the smaller mass of $\mathrm{F}$ in the HN model.

It is true that $\nu$-cross-sections contain some uncertainties. Nevertheless, the trend of $[\mathrm{F} / \mathrm{Fe}, \mathrm{O}, \mathrm{Ne}]$ discussed above is robust for these uncertainties. For the galactic chemical evolution calculation using these yields, see Kobayashi et al. (2011).

\section{References}

Heger, A., Kolbe, E., Haxton, W. C., et al. 2005, Phys. Lett. B. 606, 258

Izutani, N., Umeda, H., \& Tominaga, N. 2009, ApJ, 692, 1517

Izutani, N. \& Umeda, H. 2010, ApJ, 720, L1

Kobayashi, C., Izutani, N., Karakas, A. I., Yoshida, T., Yong, D., \& Umeda, H. 2011, ApJ, 739, L57

Nakamura, K., Yoshida, T., Shigeyama, T., \& Kajino, T. 2010, ApJ, 718, L137

Umeda, H. \& Nomoto, K., 2002, ApJ, 565, 385

Woosley, S. E., Hartmann, D. H., Hoffman, R. D., \& Haxton, W. C. 1990, ApJ, 356, 272

Woosley, S. E. \& Weaver, T. A. 1995, ApJS, 101, 181

Yoshida, T., Terasawa, M., Kajino, T., \& Sumiyoshi, K. 2004 ApJ, 600, 204

Yoshida, T., Umeda, H., \& Nomoto, K. 2008, ApJ, 672, 1043 